\title{
Influence of Thymoquinone Exposure on the Micro-Hardness of Dental Enamel: An In Vitro Study
}

\author{
Imran Farooq ${ }^{1}$ Saqib Ali ${ }^{1, \odot}$ Intisar Ahmad Siddiqui ${ }^{2}$ \\ ${ }^{1}$ Department of Biomedical Dental Sciences, College of Dentistry, \\ Imam Abdulrahman Bin Faisal University, Dammam, Saudi Arabia \\ 2Department of Quality Assurance, College of Dentistry, Imam \\ Abdulrahman Bin Faisal University, Dammam, Saudi Arabia \\ ${ }^{3}$ Department of Preventive Dental Sciences, College of Dentistry, \\ Imam Abdulrahman Bin Faisal University, Dammam, Saudi Arabia \\ ${ }^{4}$ Department of Physiology, College of Medicine, Imam \\ Abdulrahman Bin Faisal University, Dammam, Saudi Arabia
}

\author{
Khalifa S. Al-Khalifa ${ }^{3} \quad$ Mohammed Al-Hariri ${ }^{4}$
}

Eur J Dent 2019;13:318-322

\begin{abstract}
Address for correspondence Saqib Ali, BDS, MFGDP, MSc, Department of Biomedical Dental Sciences, College of Dentistry, Imam Abdulrahman Bin Faisal University, Dammam 31441, Kingdom of Saudi Arabia (e-mail: drsaqiibali@gmail.com).
\end{abstract}

\begin{abstract}
Objective The aim of this study was to assess changes in micro-hardness level of enamel after it was exposed to thymoquinone (TQ).

Materials and Methods Sixteen enamel blocks were prepared and divided into two groups (each group received eight blocks, $n=8$ ); Gp 1 (control): enamel blocks kept in $100 \mathrm{~mL}$ artificial saliva (AS) for 24 hours and Gp 2: enamel blocks kept in a mixture of TQ powder $(1 \mathrm{~g})$ and AS $(100 \mathrm{~mL})$ for 24 hours. Post-immersion they were subjected to simulated brushing with each sample receiving 8,000 linear strokes. For brushing, $3 \mathrm{~mL}$ of AS and TQ oil was used for groups 1 and 2, respectively. Enamel surfaces were analyzed for changes in values of surface micro-hardness (pre-immersion, post-immersion, and post-brushing) by obtaining Vickers hardness number (VHN).

Results The present study indicated improvement in micro-hardness levels for both groups although experimental group showed more enhancement. The mean baseline VHN for control group was 498.6, 500.4 for post-immersion, and 503.5 for post-brushing. The mean baseline VHN for experimental group was 448.7, 531 for post-immersion, and 610.3 for post-brushing. Statistically significant differences $(p<0.05)$ were observed when post-brushing VHN values of both groups were compared and also within the experimental group when post-brushing values were compared with baseline values.

Keywords

- enamel

- micro-hardness

- Nigella sativa

- thymoquinone

Statistical Analysis Wilcoxon signed-rank test was applied for the evaluation of preand post-exposure hardness values. Level of significance was $\leq 0.05$.

Conclusion The exposure of enamel to TQ led to an improvement in its micro-hardness levels. Further studies are required to understand the mechanism of action of TQ on human tissues.
\end{abstract}

\section{Introduction}

Human oral mouth is a host to around 700 bacterial species $^{1}$ living in equilibrium. A disease only occurs when there is a disturbance in this equilibrium and the balance shifts toward pathogenic species. ${ }^{2}$ Human tooth is made up of three hard tissues (enamel, dentin, and cementum). Enamel that covers the anatomical crown of the tooth and is hardest among these tissues. It covers only the dentin in the crown, and dentin in the root is covered by a thin layer of calcified cementum. Enamel is a highly mineralized hard tissue that is $4 \mathrm{wt} \%$ organic and $96 \mathrm{wt} \%$ inorganic in composition. The presence 
of high inorganic content makes it the hardest tissue in the human body. Enamel once lost cannot be regenerated or biologically repaired. ${ }^{3}$ Dental caries initiates from the surface of the enamel as it is clinically exposed to oral environment, ${ }^{4}$ and the microbes and oral plaque play an important role in its etiology. ${ }^{5}$

Dental caries is one of the most prevalent diseases and is caused by destruction of tooth structure by acids produced from cariogenic bacteria after they ferment dietary carbohydrates. ${ }^{6}$ Streptococcus mutans are gram positive bacteria that are usually found in biofilms attached to surfaces of the teeth. ${ }^{7}$ Streptococcus mutans is considered the major cariogenic species because of its ability to produce large amounts of acids, thus surpassing noncariogenic organisms and causing demineralization of hard tissues of teeth..$^{8,9}$ When the demineralization continues, it causes cavitation that provides cariogenic bacteria with a protected space in which dental caries can grow progressively. ${ }^{10}$

Nigella sativa is a medicinal plant also commonly called black seed, black cumin, and/or Habbatul Barakah. ${ }^{11}$ It is used extensively by millions of Muslims around the world and has a religious significance attached to its use ${ }^{12}$ Historically, Nigella sativa seeds have been used for the treatment of body aches, nose congestion, and intestinal worms. ${ }^{13}$ These seeds are considered to be an active source of providing ions like calcium, ${ }^{14}$ which could be useful in remineralization of tooth structure.

One of the active components of Nigella sativa is thymoquinone (TQ) that has been reported to be anticarcinogenic, ${ }^{15}$ anti-inflammatory, analgesic, and antimicrobial. ${ }^{16}$ TQ has also shown promising results in a previous animal study where Shaker and Al-Wafi evaluated TQ for the prevention of gingival inflammation in a rat model. ${ }^{17}$ It was concluded that gingival inflammation and periodontal indices were significantly lower in rats treated with TQ as compared with the control groups. The role of TQ in healing oral ulcers is also present in the literature. ${ }^{18}$ Al-Thobity et al demonstrated in a previous study that the addition of TQ in acrylic denture base could be useful in preventing adhesion of Candida albicans to it, thus resulting in prevention of denture stomatitis. ${ }^{19}$

There is a deficit of literature dealing with the effects of TQ on the hardness of dental enamel. Therefore, the aim of the present study was to evaluate changes in the micro-hardness of enamel after it is exposed to TQ (by simple immersion and by simulated tooth brushing).

\section{Materials and Methods}

Ethical approval was obtained from the Scientific Research Unit of the institute (Ref: EA 2018001) and all the ethical protocols were strictly followed. Dry powder extract of TQ (Sigma-Aldrich) and TQ oil was obtained and used in the experiments.

\section{Artificial Saliva Preparation}

Artificial saliva (AS) was prepared by mixing $\mathrm{NaCl}: 0.400$ g, KCl: 0.400 g, $\mathrm{NaH}_{2} \mathrm{PO} 4 . \mathrm{H}_{2} \mathrm{O}: 0.69 \mathrm{~g} ; \mathrm{CaCl}_{2} . \mathrm{H}_{2} \mathrm{O}: 0.795 \mathrm{~g}$;
$\mathrm{Na}_{2}$ S. $9 \mathrm{H}_{2} \mathrm{O}: 0.005 \mathrm{~g}$ in $1000 \mathrm{~mL}$ of deionized water, as suggested by Fusayama et al. ${ }^{20}$ The $\mathrm{pH}$ of freshly prepared AS was 5.5 that was adjusted to a neutral $\mathrm{pH}$ of 7.0 by adding aliquots of $1 \mathrm{M}$ of $\mathrm{NaOH}$ as proposed by Farooq et al. ${ }^{21}$

\section{Preparation of Enamel Blocks and Grouping of Specimens}

Sixteen extracted teeth $(n=16)$ were obtained from oral and maxillofacial surgery clinics of the institute. Teeth that were free from caries, restorations, or any other obvious defects were selected. Enamel blocks were prepared by cutting these teeth mesiodistally over cementoenamel junction with a water-cooled diamond saw (Isomet 5000 Linear Precision Saw; Buehler Ltd, Illinois, United States) at a blade speed of $3,000 \mathrm{rpm}$ and a feed rate of $10 \mathrm{~mm} / \mathrm{min}$. The root portion was discarded and the crown was then embedded in an acrylic block in such a manner that the buccal surface was exposed. Before the experiments, enamel surfaces were serially polished with a water-cooled carbide paper of up to 1200 grit fineness (3M; St. Paul, Minnesota, United States). These sixteen enamel blocks were then randomly divided into two groups (each group received eight blocks, $n=8$ ); Gp 1 (control): enamel blocks kept in AS for 24 hours and Gp 2: enamel blocks kept in a mixture of TQ $(1 \mathrm{~g})$ and $\mathrm{AS}(100 \mathrm{~mL})$ for 24 hours.

The mixture of AS and TQ was prepared first by weighing it using a motorized analytical balance scale (Sgm Laboratory Solutions, India) and then mixing it using a magnetic stirrer (Hot Plate stirrer LabTech, Italy).

\section{Surface Micro-Hardness Analysis}

The enamel blocks were analyzed for changes in the values of surface micro-hardness and the data were collected preand post-immersion in AS for Gp 1. For Gp 2 containing a mixture of AS + TQ the data were again collected before and after immersion in the mixture. The Vickers hardness number (VHN) values were measured using a digital micro-hardness tester (FM-ARS 9000; Future-Tech Corp, Kawasaki, Japan). In each sample, an area was marked with a nail varnish and three indents were made inside that marked area using a Vickers diamond indenter under a 100-g load applied for 10 seconds.

\section{Simulated Tooth Brushing with Thymoquinone}

Post-immersion, enamel blocks belonging to groups 1 and 2 were subjected to simulated brushing that was performed inside a toothbrush simulation machine (Toothbrush simulator; model ZM-3.8, Germany), under a load of $250 \mathrm{~g}$ for 2,000 linear strokes per day for 4 days (each sample received 8,000 strokes that is equivalent to 5 months of in vivo brushing). ${ }^{6}$ For brushing of group 1 specimens, $3 \mathrm{~mL}$ of AS and for group 2, $3 \mathrm{~mL}$ of TQ oil were used. After every brushing cycle, all the specimens were washed with distilled water thoroughly. After fourth day of brushing, specimens were washed with distilled water and were left to air dry prior to surface micro-hardness analysis again. 


\section{Statistical Analysis}

Data analysis was performed by using SPSS-20.0 (IBM product; Chicago, Illinois, United States). Wilcoxon signed-rank test was applied to evaluate significance of mean within the control and experimental groups comparing baseline values versus post-immersion and post-brushing values. A $p$-value $\leq 0.05$ was considered statistically significant difference of means.

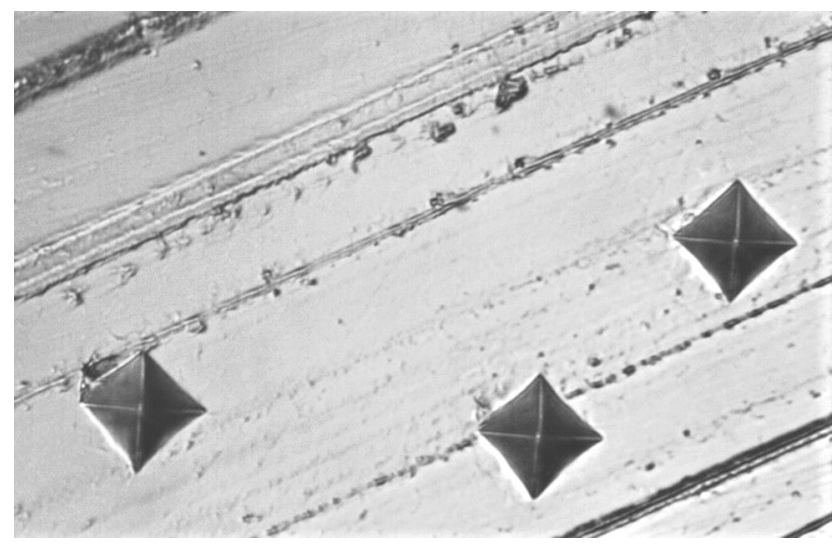

Fig. 1 Image showing indentations performed on enamel surfaces to obtain Vickers hardness number values.

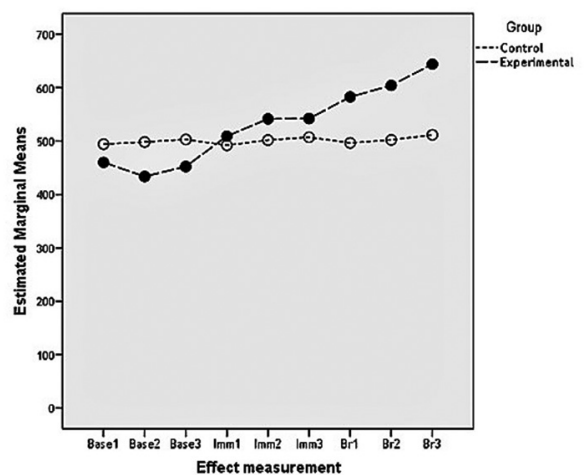

Fig. 2 Image showing a comparison of Vickers hardness number values between the two groups at baseline, post-immersion, and post-brushing.

\section{Results}

The micro-hardness level of enamel surfaces was assessed by Vickers indentation (-Fig. 1). The results of the present study indicated improvement in micro-hardness levels for both groups. For the control group in which enamel blocks were only exposed to AS, the improvement was less than the experimental group in which enamel blocks were exposed to a mixture of AS + TQ (-Fig. 2). The mean baseline VHN for the control group was 498.6 that improved to 500.4 post-immersion, and 503.5 post-brushing. The mean baseline VHN for the experimental group was 448.7 that improved to 531 post-immersion, 610.3 post-brushing (-Table 1). Statistically significant differences $(p<0.05)$ were perceived when post-brushing VHN values of both groups were compared and were also observed within the experimental group when post-brushing values were compared with baseline values.

\section{Discussion}

The results of our study show improved hardness level of enamel blocks when they were exposed to TQ (post-immersion and post-brushing), whereas, in the control group containing AS, there was only a slight improvement in the hardness level. Dental enamel is a mineralized tooth tissue that is mostly inorganic ( $96 \mathrm{wt} \%$ ) and forms the outer protective covering of teeth. ${ }^{22}$ The enamel is exposed to various acidic and masticatory challenges throughout its existence; therefore, it is important to analyze hardness levels of enamel and how they are affected by various elements. ${ }^{23,24}$

Human saliva could act as a medium and also as a chief source of transporting essential ions like calcium and phosphate to the tooth structure. ${ }^{25}$ However, the role played only by saliva in hardening of dental enamel is debatable. Enamel is open to an active oral environment and it has the ability to adsorb minerals from its environment by mechanism of simple diffusion. ${ }^{26}$ Alencar et al performed an in situ study to understand hardening of acid-softened enamel by saliva at different time intervals and concluded that it takes at least 2 hours to achieve partial hardening of enamel only. ${ }^{27}$ In another similar in vitro study, Wang et al reported that application of AS for several hours could only lead to limited recovery of apatite

Table 1 Mean VHN for both groups measured at baseline, post-immersion, and post-brushing

\begin{tabular}{|l|l|l|l|}
\hline Groups & Control group (mean \pm SD) & $\begin{array}{l}\text { Experimental group } \\
\text { (mean } \pm \text { SD) }\end{array}$ & $p$-Value \\
\hline Baseline & $498.63 \pm 83.85$ & $448.73 \pm 103.39$ & 0.307 \\
\hline Post-immersion & $500.46 \pm 85.89$ & $531.02 \pm 104.86$ & 0.534 \\
\hline Post-brushing & $503.50 \pm 85.65$ & $610.36 \pm 68.67^{\mathrm{a}, \mathrm{b}}$ & 0.016 \\
\hline
\end{tabular}

Abbreviations: AS, artificial saliva; SD, standard deviation; TQ, thymoquinone; VHN, Vickers hardness number.

Note: Baseline: Mean VHN of eight enamel blocks after three indentations in each sample for both the groups, Post-immersion: Mean VHN of eight enamel blocks after three indentations each sample in both the groups post-24 hour exposure to AS and TQ for group 1 and 2 respectively, Post-brushing: Mean VHN of eight enamel blocks after three indentations in each sample for both the groups post simulated brushing with AS and TQ for groups 1 and 2 respectively.

Wilcoxon sign-rank test.

${ }^{a}$ Significant mean difference between control and experimental groups.

bSignificant mean difference within the group baseline versus post brushing. 
enamel structure. ${ }^{28}$ Our study revealed similar results where enamel hardness was only slightly increased after exposure to AS, although in our study enamel blocks were not demineralized before being exposed to AS and to the mixture of AS + TQ.

It is noteworthy to mention that even after stirring the mixture for 2 hours in our study, TQ was only partially dissolved in AS. This could be attributed to the physicochemical nature of TQ that is identical to hydrophobic materials that may well lead to its poor bioavailability. ${ }^{29}$ Nevertheless, the mixture of AS + TQ showed significantly higher micro-hardness levels of enamel surface post-immersion. TQ is chemically 2-methyl-5-isopropyl-1, 4-benzoquinone. ${ }^{30}$ It is the main bioactive component of Nigella sativa and TQ could be accountable for its main biological actions. ${ }^{31}$ Many researchers also believe that all benefits of Nigella sativa are actually due to the presence of TQ in its composition. ${ }^{32-37}$

Post-brushing, micro-hardness values of the experimental group were significantly increased. It could not be said with authority what led to an increase in micro-hardness level of enamel surfaces in our study but the only variable changed between the groups was brushing enamel blocks with TQ oil in the experimental group. This could have possibly improved the hardness of enamel surfaces in that group.

One limitation of our study is its in vitro nature as actual in vivo conditions but to overcome this, every attempt was made to standardize all the variables (teeth number of both groups, exposure time, quantity of exposed solution). Another limitation of our study was that it is almost impossible to take baseline, post-immersion, and post-brushing hardness values from exactly the same area of enamel's surface. To minimize the variation, the area from that we aimed to take hardness values was marked first and then micro-hardness was analyzed in only that particular area. The same area was brushed during simulated brushing with AS and TQ for our control and experimental groups. In the future, comparison of TQ with a fluoride containing toothpaste could prove useful to determine efficacy of both products in remineralizing enamel.

To the best of the author's knowledge, this is the first in vitro study that has tested TQ's effect on the micro-hardness level of dental enamel. The positive results of our study could lead to future in vivo studies to understand TQ's effect in a more vigorous dynamic oral environment.

\section{Conclusion}

Exposure to TQ led to an increased micro-hardness of enamel surfaces as compared with the enamel blocks exposed to AS only. Future clinical studies are indicated to test TQ under intense in vivo oral conditions.

\section{Authors' Contributions}

All authors have made substantive contribution to this study and/or manuscript, and all have reviewed the final paper prior to its submission.

\section{Conflict of Interest}

None declared.

\section{Acknowledgments}

The authors are grateful to Vice Deanship of Post-Graduate Studies and Research at the College of Dentistry, Imam Abdulrahman Bin Faisal University, Dammam, Saudi Arabia for facilitating us with the micro-hardness analysis.

\section{References}

1 Dewhirst FE, Chen T, Izard J, et al. The human oral microbiome. J Bacteriol 2010;192(19):5002-5017

2 Kuboniwa M, Tribble GD, Hendrickson EL, Amano A, Lamont RJ, Hackett M. Insights into the virulence of oral biofilms: discoveries from proteomics. Expert Rev Proteomics 2012;9(3):311-323

3 Zafar MS, Ahmed N. Nano-mechanical evaluation of dental hard tissues using indentation technique. World Appl Sci J 2013;28(10):1393-1399

4 Zafar MS, Ahmed N. The effects of acid etching time on surface mechanical properties of dental hard tissues. Dent Mater J 2015;34(3):315-320

5 Alfaroukh R, ElEmbaby A, Almas K, et al. Oral biofilm formation and retention on commonly used dental materials: an update. Odontostomatol Trop 2018;41(164):28-34

6 Alhussain AM, Alhaddad AA, Ghazwi MM, Farooq I. Remineralization of artificial carious lesions using a novel fluoride incorporated bioactive glass dentifrice. Dent Med Probl 2018;55(4):379-382

7 Zero DTFM, Fontana M, Martínez-Mier EA, et al. The biology, prevention, diagnosis and treatment of dental caries: scientific advances in the United States. J Am Dent Assoc 2009;140(Suppl 1):25S-34S

8 Lemos JA, Quivey RG, Jr. Koo H, Abranches J. Streptococcus mutans: a new gram-positive paradigm? Microbiology 2013;159(Pt 3):436-445

9 Raja M, Hannan A, Ali K. Association of oral candidal carriage with dental caries in children. Caries Res 2010;44(3):272-276

10 Rouabhia M, Chmielewski W. Diseases associated with oral polymicrobial biofilms. Open Mycol J. 2012;6:27-32

11 Al-Attass SA, Zahran FM, Turkistany SA. Nigella sativa and its active constituent thymoquinone in oral health. Saudi Med J 2016;37(3):235-244

12 Rahmani AH, Aly SM. Nigella Sativa and its active constituents thymoquinone shows pivotal role in the diseases prevention and treatment. Asian J Pharm Clin Res. 2015;8(1):48-53

13 Gaur S, Shrivastava B, Gaur S, Bhardwaj R, Khanchandani R. Medicinal and therapeutical potential of Nigella sativa. Int J Med App Sci Res 2014;1:32-39

14 Ahmad A, Husain A, Mujeeb M, et al. A review on therapeutic potential of Nigella sativa: a miracle herb. Asian Pac J Trop Biomed 2013;3(5):337-352

15 Padhye S, Banerjee S, Ahmad A, Mohammad R, Sarkar FH. From here to eternity - the secret of Pharaohs: therapeutic potential of black cumin seeds and beyond. Cancer Ther 2008;6b: 495-510

16 Ahmad I, Tripathi J, Sharma M, Karchulli MS, Umer L. Nigella sativa - a medicinal herb with immense therapeutic potential (a systematic review) Int J Bio Pharm Res 2014;5:755-762

17 Shaker A, Al-Wafi H. Benefits of thymoquinone, a Nigella Sativa extract in preventing dental caries initiation and improving gingival health. ProQuest 2014;LLC:72 
18 Al-Douri A, Al-Kazaz S. The effect of Nigella Sativa oil (black seed) on the healing of chemically induced oral ulcer in rabbit (experimental study) Al-Rafidain Dent J 2010;10:151-157

19 Al-Thobity AM, Al-Khalifa KS, Gad MM, Al-Hariri M, Ali AA, Alnassar T. In vitro evaluation of the inhibitory activity of thymoquinone in combatting Candida albicans in denture stomatitis prevention. Int J Environ Res Public Health 2017;14(7):743

20 Fusayama T, Katayori T, Nomoto S. Corrosion of gold and amalgam placed in contact with each other. J Dent Res 1963;42:1183-1197

21 Farooq I, Moheet IA, AlShwaimi E. In vitro dentin tubule occlusion and remineralization competence of various toothpastes. Arch Oral Biol 2015;60(9):1246-1253

22 Zafar MS. A comparison of dental restorative materials and mineralized dental tissues for surface nanomechanical properties. Life Sci J 2014;1110s :19-24

23 Gutiérrez-Salazar M, Reyes-Gasga J. Microhardness and chemical composition of human tooth. Mater Res 2003;6(3):367-373

24 Hassan U, Farooq I, Moheet IA, AlShwaimi E. Cutting efficiency of different dental materials utilized in an air abrasion system. Int J Health Sci (Qassim) 2017;11(4):23-27

25 Shaikh K, Pereira R, Gillam DG, Phad S. Comparative evaluation of desensitizing dentifrices containing BioMin ${ }^{\circledR}$, Novamin ${ }^{\circledR}$ and fluoride on dentinal tubule occlusion before and after a citric acid challenge- a scanning electron microscope in-vitro Study. J Odontol 2018;2(1):105

26 Zafar MS, Ahmed N. Nanomechanical characterization of exfoliated and retained deciduous incisors. Technol Health Care 2014;22(6):785-793

27 Alencar CR, Mendonça FL, Guerrini LB, et al. Effect of different salivary exposure times on the rehardening of acid-softened enamel. Braz Oral Res 2016;30(1):e104
28 Wang X, Mihailova B, Klocke A, Heidrich S, Bismayer U. Effect of artificial saliva on the apatite structure of eroded enamel. Int J Spectrosc 2011; 2011. Available at: http://www.hindawi. com/journals/ijs/2011/236496/.doi:10.1155/2011/236496

29 Lebwohl M, Ali S. Treatment of psoriasis. Part 2. Systemic therapies. J Am Acad Dermatol 2001;45(5):649-661, quiz 662-664

30 Kishwar F, Mahmood T, Mahmood I, Anwar A, Parween R, Mustafa S. Complexation of active ingredient thymoquinone of nigella sativa (black seed) with chromium(vi) Fuuast J BIOL. 2016;6(1):65-72

31 Ermumcu MSK, Sanher N. Black cumin (Nigella sativa) and its active component of thymoquinone: effects on health. J Food Health Sci 2017;3(4):170-183

32 Mehta BK, Pandit V, Gupta M. New principles from seeds of Nigella sativa. Natural product research. Part A 2009;23:138-148

33 Akram Khan M, Afzal M. Chemical composition of Nigella sativa Linn: Part 2 recent advances. Inflammopharmacology 2016;24(2)(3):67-79

34 Khazdair MR. The protective effects of Nigella sativa and its constituents on induced neurotoxicity. J Toxicol 2015;2015:841823

35 Beheshti F, Khazaei M, Hosseini M. Neuropharmacological effects of Nigella sativa. Avicenna J Phytomed 2016;6(1):104-116

36 Darakhshan S, Bidmeshki Pour A, Hosseinzadeh Colagar A, Sisakhtnezhad S. Thymoquinone and its therapeutic potentials. Pharmacol Res 2015;95-96:138-158

37 El-Dakhakhny M, Madi NJ, Lembert N, Ammon HP. Nigella sativa oil, nigellone and derived thymoquinone inhibit synthesis of 5-lipoxygenase products in polymorphonuclear leukocytes from rats. J Ethnopharmacol 2002;81(2):161-164 